\title{
Near-Infrared Photothermal Therapy Cancer Treatment Assisted with Graphene-Based Materials ${ }^{\dagger}$
}

\author{
Faith Cheung 1,* \\ 1 Richard Montgomery High School, USA \\ * Correspondence: faithrmhs@gmail.com (F.C.); \\ $\dagger$ Presented at Materials Chemistry and Physics (Materials Chemistry 2020) - International e-Conference
}

Received: 16.09.2020; Revised: 20.09.2020; Accepted: 24.09.2020; Published: 27.09.2020

\begin{abstract}
Photothermal therapy is an emerging method of cancer treatment in which tumors are ablated by heating agents using near-infrared light (700-1000 nm). A semiconductor with a bandgap between 0.3-0.7 eV would, therefore, efficiently emit near-infrared light. The new "magic" material graphene has a bandgap of zero, which is advantageous with regard to designing a new material with a suitable bandgap for the emission of near-infrared light. In our investigations, using the first-principles density functional theory calculation method, we aimed to and successfully designed graphene-based materials with a direct bandgap of $0.68 \mathrm{eV}$. They have the potential to be optimal and efficient near-infrared light sources due to their narrow yet fitting bandgap. The present results open up a new avenue for the application of graphene-based materials to assist in photothermal therapy.
\end{abstract}

Keywords: biomedical engineering; materials chemistry; materials science; oncology; physical chemistry; photothermal therapy; bandgap; graphene; first principle calculation.

(C) 2020 by the authors. This article is an open access article distributed under the terms and conditions of the Creative Commons Attribution (CC BY) license (https://creativecommons.org/licenses/by/4.0/).

\section{Funding}

This research received no external funding.

\section{Acknowledgments}

This research has no acknowledgment.

\section{Conflicts of Interest}

The authors declare no conflict of interest. 